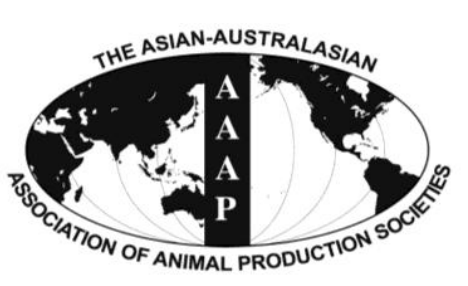

\title{
Effects of Feeding Rate and Water Temperature on Growth and Body Composition of Juvenile Korean Rockfish, Sebastes schlegeli (Hilgendorf 1880)
}

\author{
Rahman Md Mizanur ${ }^{1,3}$, Hyeonho Yun ${ }^{1}$, M Moniruzzaman ${ }^{1}$, F Ferreira ${ }^{1}$, Kang-woong Kim ${ }^{2}$, and Sungchul C Bai ${ }^{1, *}$ \\ ${ }^{1}$ Department of Marine Bio-Materials and Aquaculture/Feeds and Foods Nutrition Research Center, \\ Lab 9134B/C-21, Pukyong National University, Busan 608-737, Korea
}

\begin{abstract}
Three feeding trials were conducted to evaluate the effects of feeding rate and water temperature on growth and body composition of juvenile Korean rockfish, Sebastes schlegeli rearing at 3 different water temperatures. A total of 270 fish (each experiment) individual body weight (BW) averaging $16 \pm 0.3 \mathrm{~g}$ (mean $\pm \mathrm{SD}$ ) were fed a commercial diet for $4 \mathrm{wk}$ at $16^{\circ} \mathrm{C}, 20^{\circ} \mathrm{C}$, and $24^{\circ} \mathrm{C}$. At each temperature, triplicate tanks were assigned to one of 6 feeding rates: $1.5 \%, 2.5 \%, 2.8 \%, 3.1 \%, 3.4 \%$, and satiation $(3.7 \%$ $\mathrm{BW} / \mathrm{d})$ at $16^{\circ} \mathrm{C}, 1.9 \%, 2.9 \%, 3.2 \%, 3.5 \%, 3.8 \%$ and satiation $(4.1 \% \mathrm{BW} / \mathrm{d})$ at $20^{\circ} \mathrm{C}$ and $1.7 \%, 2.7 \%, 3.0 \%, 3.3 \%, 3.6 \%$, and satiation $(3.9 \% \mathrm{BW} / \mathrm{d})$ at $24^{\circ} \mathrm{C}$ water temperature. Weight gains of fish in satiation and $3.4 \%$ groups at $16^{\circ} \mathrm{C}$, in satiation and $3.8 \%$ groups at $20^{\circ} \mathrm{C}$ and in satiation and $3.6 \%$ groups at $24^{\circ} \mathrm{C}$ were significantly higher than those of fish in the other treatments $(\mathrm{p}<0.05)$. A broken line regression analysis of weight gain indicated that optimum feeding rates of juvenile Korean rockfish were $3.41 \%$ at $16^{\circ} \mathrm{C}, 3.75 \%$ at $20^{\circ} \mathrm{C}$ and $3.34 \%$ at $24^{\circ} \mathrm{C}$ water temperature. Results of the present study indicate that the optimum feeding rate could be $>3.1 \%$ but $<3.41 \%$ at $16^{\circ} \mathrm{C},>3.5 \%$ but $<3.75 \%$ at $20^{\circ} \mathrm{C}$ and $>3.0 \%$ but $<3.34 \%$ at $24^{\circ} \mathrm{C}$. As we expected results suggest that fish performed better at $20^{\circ} \mathrm{C}$ than $16^{\circ} \mathrm{C}$ or $24^{\circ} \mathrm{C}$ water temperature and the optimum feeding rate could be $3.1 \% \mathrm{BW} / \mathrm{d}$ to $3.7 \%$ BW/d in $16 \mathrm{~g}$ of juvenile Korean rockfish. (Key Words: Juvenile Korean Rockfish, Sebastes schlegeli, Feeding Rate, Water Temperature, Growth Performance)
\end{abstract}

\section{INTRODUCTION}

Each fish species has an optimum water temperature range in which the fish can grow fast. Water temperature is a major influence on aquaculture husbandry practices and it has also a profound impact on the feeding rate. Feeding rate, water temperature, and fish size are 3 important factors which are synergistically affecting the growth of fish (Gardeur et al., 2007). All fish species are characterized by

\footnotetext{
* Corresponding Author: Sungchul C. Bai. Tel: +82-51-629-7922, Fax: +82-51-628-6873, E-mail: scbai@pknu.ac.kr

2 Aquafeed Research Center, East Sea Fisheries Research Institute, National Fisheries Research and Development Institute (NFRDI), Pohang 791-802, Korea.

3 Bangladesh Civil Service Cadre, Planning Commission, Ministry of Planning, Dhaka, Bangladesh.

Submitted Aug. 15, 2013; Revised Nov. 17, 2013; Accepted Jan. 8, 2014
}

an ideal range of temperature in which they show their maximum growth (Person-Le et al., 2006; Bjornsson et al., 2007; Oyugi et al., 2011). Several studies have been reported that the specific water temperature range showed the faster growth in Pikeperch, Sander lucioperca at $20^{\circ} \mathrm{C}$ to $25^{\circ} \mathrm{C}$ (Molnar et al., 2006; Ronyai and Csengeri, 2008; Schulz et al., 2008). Low temperature causes sluggishness by retarding the digestion speeding of fish (Bailey and Alanara, 2006). Some researchers have found that the digestion rate has been increased as the temperature increases (Turker, 2009). Environmental temperature is one of the most important ecological factor which also influence the behavior and physiological process of aquatic animals (Xia and Li, 2010).

Optimal feeding rate is the most important factor to success of any aquaculture operation. And it is particularly appropriate for the juvenile fish because of their 
susceptibility to overfeeding and underfeeding which causes both increased incidences of disease and mortality. Deng et al. (2003) investigated the optimal feeding rate from larvae to yearlings based on their growth performance. However, the optimal feeding rate depends on fish size and water temperature (Kestemont and Barras, 2001; Wang et al., 2009). Maximum growth was found in pike perch, Sander lucioperca when feed was delivered at the satiation status (Nyina-Wamwiza et al., 2005; Schulz et al. 2008). Hung et al. (1993) reported the effects of feeding rate and water temperature on the growth performance in juvenile white sturgeon, Acipenser transmontanus. Optimum feeding rate is regularly impeded by environmental pollution and unnecessary feed losses which are more efficient for fish growth. When the fish is fed inadequate or over, feed efficiency and growth can decrease and this situation increases the cost of fish production as well as the water quality may deteriorate for over feeding (Dwyer et al., 2002). Optimum feeding rate and feeding frequency are depending on fish species, size and rearing system (Cho et al., 2003).

Korean rockfish, Sebastes schlegeli (Hilgendorf) inhabits shallow rocky shores along the all coasts of the Korean Peninsula, Japan, and some parts of China. It is one of the most important commercial marine fish species in Korean Peninsula and commercial farming of this fish has been developed since 1987 (Bai et al., 2001). Korean rock fish has several important characteristics for aquaculture such as good tolerance to low water temperature, fast growth and higher survival rate. Korean rockfish are mainly cultured in net cages, although some are raised in ponds (Bai and Okorie, 2009). Feeding rate should be optimal, as the fish can get enough feed and represent the maximum growth rate. There is limited information available concerning the optimum feeding rates and water temperatures for maximum growth of this fish. Therefore the objective of this study was to determine the effects of feeding rate on growth performance and body composition in juvenile Korean rockfish rearing at 3 different water temperatures.

\section{MATERIALS AND METHODS}

\section{Experimental diet}

Commercial feed was used in all the feeding trials which was supplied by Suhyup Feed Company Limited (Uiryeong, Gyeongsangnamdo, Korea). The commercial diets extruded pellet (EP) size was 2.4 to $2.6 \mathrm{~mm}$ and the feed contained $46.61 \%$ crude protein, $11.06 \%$ crude lipid, $13.94 \%$ crude ash and $8.60 \%$ moisture.

\section{Experimental fish and feeding trials}

Juvenile Korean rockfish were collected in July, 2012 from a hatchery of Tongyeong coastal city and they were transported to the Department of Fisheries Biology of Pukyong National University, Busan, Korea. Prior to start of the experiment, fish were acclimated with the experimental diet and conditions. After acclimatization, three aquaria were selected to determine the satiation. Fish were fed 3 times a day to apparent the satiation for a week. For satiation, fish were fed as much as they could ingest at each feeding time. Feeding was done by hand very slowly and carefully to ensure ingestion of feed completely. Finally the remaining feed weight was subtracted from the initial weight and calculated as the percentage of satiation.

The seawater temperature was maintained at $16^{\circ} \mathrm{C}$, $20^{\circ} \mathrm{C}$, and $24^{\circ} \mathrm{C}$ by using both water heater and a cooling machine in the center tank during the whole experimental period. These 3 water temperatures were selected based on annual seasonal natural water temperature of the Korean sea for the different seasons recorded annually. After $1 \mathrm{wk}$ of satiation, 270 fish (Each experiment) with initial individual $\mathrm{BW}$ of $16.2 \pm 0.13 \mathrm{~g}$ (mean $\pm \mathrm{SD}$ ) for $16^{\circ} \mathrm{C}$ experiment, 16.3 $\pm 0.11 \mathrm{~g}$ (mean $\pm \mathrm{SD})$ for $20^{\circ} \mathrm{C}$ experiment and $16.1 \pm 0.13 \mathrm{~g}$ (mean $\pm \mathrm{SD}$ ) for $24^{\circ} \mathrm{C}$ experiment were randomly distributed into each of 18 aquaria. Each aquarium was then randomly assigned to one of the triplicates of the 6 respective feeding rates $1.5 \%, 2.5 \%, 2.8 \%, 3.1 \%, 3.4 \%$, and satiation $(3.7 \%$ $\mathrm{BW} / \mathrm{d})$ in the experiment of $16^{\circ} \mathrm{C}$ and $1.9 \%, 2.9 \%, 3.2 \%$, $3.5 \%, 3.8 \%$, and satiation $(4.1 \% \mathrm{BW} / \mathrm{d})$ in the experiment of $20^{\circ} \mathrm{C}$ and $1.7 \%, 2.7 \%, 3.0 \%, 3.3 \%, 3.6 \%$, and satiation $(3.9 \% \mathrm{BW} / \mathrm{d})$ in the experiment of $24^{\circ} \mathrm{C}$ water temperature.

The feeding trial was conducted by using a semirecirculation system with $40-\mathrm{L}$ rectangular tanks receiving filtered seawater which flow rate of around $2 \mathrm{~L} / \mathrm{min}$ from the central tank. Supplemental aerations were provided to maintain dissolved oxygen levels near $6.5 \pm 0.5 \mathrm{mg} / \mathrm{L}$ and the salinity of used sea water was about $33 \pm 1$ ppt in all the feeding trials. Total BW in each aquarium was determined every $2 \mathrm{wk}$, and the amounts of diet fed were adjusted accordingly to the increasing $\mathrm{BW}$ in each trial. Fish were fed thrice a day at 08:00, 14:00, and 20:00 hours with each daily ration divided into three portions by hand with the satiation level which was followed by Wang et al. (2006). In order to maintain a good environmental condition siphoning was carried out twice a day and within every 2 days water was changed from the system. During the event of water replacement, temperature was maintained slowly from the normal temperature to desired temperature by using a water heater and cooling machine to avoid unnecessary stress of fish.

\section{Sample collection and analyses}

After 4 wk of feeding trial, fish were kept starved for 24 $\mathrm{h}$ and they were counted and weighed. Weight gain (WG), specific growth rate (SGR), feed efficiency (FE), protein 
efficiency ratio (PER), survival and whole body proximate composition were measured and calculated. At the end of feeding trial, total length and total BW were measured five randomly selected juvenile Korean rockfish fish from each tank. And those five fish were dissected and measured their liver and visceral weight to determine the biological indices such as condition factor (CF), hepatosomatic index (HSI), and visceral somatic index (VSI). Blood samples were taken by using a heparinized syringes from the caudal vein of five randomly chosen fish per tank and then blood analysis was performed to get some parameters. Blood serum was collected after centrifugation $(3,000 \mathrm{rpm}$ for 10 $\min$ ) and stored at $-70^{\circ} \mathrm{C}$ in order to analyze the hematocrit (HCT), hemoglobin (Hb), glutamic oxaloacetic transaminase (GOT) and glutamic pyruvic transaminase (GPT). Hematocrit and hemoglobin were measured by using the Drabkin's reagent method (Brown, 1980). Blood serum analyses were performed by the National Fisheries Research and Development Institute (NFRDI), Gijang-Gun, Busan, Korea. Blood serum was analyzed in NFRDI by using the kits of DRI-CHEM 4000i- Fuji Dri-Chem Slide3150 (Minato-ku, Tokyo, Japan). Rest five fish from each tank were used to analyze the whole-body proximate composition. Proximate composition analyses of experimental diets and fish body were performed by the standard methods of AOAC (1995). To determine the moisture content of diets and fish were dried to maintain constant weights at $105^{\circ} \mathrm{C}$ for $24 \mathrm{~h}$. Ash content was determined using a muffle furnace $\left(550^{\circ} \mathrm{C}\right.$ for $\left.4 \mathrm{~h}\right)$. Crude lipid content was determined by the soxhlet extraction method by using Soxtec system 1046 (Foss, Hoganas, Sweden) and crude protein content by Kjeldahl method $(\mathrm{N} \times 6.25)$ after acid digestion.

\section{Statistical analyses}

All data were analyzed by one way analysis of variance (ANOVA) test using Computer Program Statistics 3.1 (Analytical Software, St. Paul, MN, USA) to test for the dietary treatments. ANOVA used for the treatments of continuous variable. Treatment effects were considered with the significant level at $\mathrm{p}<0.05$. Broken-line regression model was used to analyze the weight gain of juvenile Korean rockfish in response to optimum feeding rate (Robbins et al., 1979).

The effects of feeding rates on growth performance and body composition were assessed by the following formulae:

\footnotetext{
Satiation (SA: \% BW/d)

$=$ Total feed weight $(\mathrm{g}) \times 100 /$ total fish weight $(\mathrm{g})$
}

Weight gain (\%)

$=($ Final weight - initial weight $) \times 100 /$ initial weight
Specific growth rate $(\% / d)$

$=\left(\log _{\mathrm{e}}\right.$ final $w t-\log _{\mathrm{e}}$ initial $\left.w t\right) \times 100 /$ days

Feed efficiency (\%)

$=$ Wet weight gain $(\mathrm{g}) \times 100 /$ dry feed intake $(\mathrm{g})$

Protein efficiency ratio $=$ Wet weight gain/protein intake

Condition factor

$=$ Fish weight $(\mathrm{g}) \times 100 /$ total length $\left(\mathrm{cm}^{3}\right)$

Hepatosomatic index

$=$ Fiver weight $(\mathrm{g}) \times 100 /$ fish weight $(\mathrm{g})$

Visceralsomatic index

$=$ Visceral weight $\times 100 /$ fish weight $(\mathrm{g})$

Pooled standard error of means $($ Pooled SEM $)=\mathrm{SD} / \sqrt{\mathrm{n}}$

Survival (\%)

$=$ Number of fish at end of experiment

/number of fish stocked $\times 100$

\section{RESULTS}

\section{Feeding rates of $16 \mathrm{~g}$ of juvenile Korean rockfish reared at $16^{\circ} \mathrm{C}$}

Biological performances are shown in Table 1. At the end of $4 \mathrm{wk}$ of feeding trials at $16^{\circ} \mathrm{C}$ experiment of Korean rockfish, weight gain and specific growth rate of fish fed groups of $3.4 \%$ and satiation $(3.7 \% \quad \mathrm{BW} / \mathrm{d})$ were significantly higher than those of fish fed groups of $1.5 \%$ and $2.5 \%$ BW/d $(\mathrm{p}<0.05)$. There were no significant differences of WG and SGR among fish fed groups at 3.4\% and satiation $(3.7 \% \mathrm{BW} / \mathrm{d})$ but WG, SGR, and FE of fish fed at $1.5 \%$ were significantly lower than those of other fish fed groups. Feed efficiency of fish fed group at 3.4\% BW/d was significantly higher than those of other fish fed groups. There were no significant difference of FE at the fish fed groups of $2.8 \%$ and satiation $(3.7 \% \mathrm{BW} / \mathrm{d})$. No significant difference was found in PER at all the fish fed groups. Survival rate of fish fed group of $1.5 \%$ was significantly lower and fish fed group of $3.4 \%$ was significantly higher than other fish fed groups. There were no significantly different of survival at the fish fed at $2.8 \%$ and satiation fed $(3.7 \% \mathrm{BW} / \mathrm{d})$. Broken line analysis of weight gain demonstrated that the optimum feeding rates of juvenile Korean rockfish was $3.41 \%(\mathrm{BW} / \mathrm{d})$ at $16^{\circ} \mathrm{C}$ water temperature (Figure 1). The results indicated that the optimum daily feeding rate of juvenile Korean rockfish could be greater than $3.4 \%$ but less than $3.7 \%(\mathrm{BW} / \mathrm{d})$ at $16^{\circ} \mathrm{C}$ water temperature. 
Table 1. Growth performance, whole-body proximate composition, morphological indices and serological characteristics of $16 \mathrm{~g}$ fish reared at $16^{\circ} \mathrm{C}$ water temperature ${ }^{1}$

\begin{tabular}{|c|c|c|c|c|c|c|c|}
\hline \multirow{2}{*}{ Item } & \multicolumn{7}{|c|}{ Diets } \\
\hline & $1.5 \%$ & $2.5 \%$ & $2.8 \%$ & $3.1 \%$ & $3.4 \%$ & $\mathrm{SA}^{2}$ & Pooled SEM \\
\hline \multicolumn{8}{|c|}{ Growth performance } \\
\hline $\mathrm{WG}^{3}$ & $41.74^{\mathrm{f}}$ & $61.72^{\mathrm{e}}$ & $70.18^{\mathrm{d}}$ & $80.86^{\mathrm{bc}}$ & $85.27^{\mathrm{ab}}$ & $87.11^{\mathrm{a}}$ & 4.09 \\
\hline $\mathrm{SGR}^{4}$ & $1.43^{\mathrm{d}}$ & $2.02^{\mathrm{c}}$ & $2.21^{\mathrm{bc}}$ & $2.47^{\mathrm{ab}}$ & $2.57^{\mathrm{a}}$ & $2.61^{\mathrm{a}}$ & 0.11 \\
\hline $\mathrm{FE}^{5}$ & $83.03^{\mathrm{d}}$ & $83.54^{\mathrm{d}}$ & $85.38^{\mathrm{b}}$ & $84.17^{\mathrm{c}}$ & $90.25^{\mathrm{a}}$ & $85.33^{\mathrm{b}}$ & 3.50 \\
\hline $\mathrm{PER}^{6}$ & 1.72 & 1.76 & 1.77 & 1.81 & 2.02 & 1.82 & 0.06 \\
\hline Survival $^{7}$ & $97.42^{\mathrm{d}}$ & $98.34^{\mathrm{b}}$ & $97.85^{\mathrm{c}}$ & $98.41^{\mathrm{b}}$ & $100^{\mathrm{a}}$ & $97.61^{\mathrm{c}}$ & 0.12 \\
\hline \multicolumn{8}{|c|}{ Whole-body proximate composition } \\
\hline Moisture & $72.74^{\mathrm{bc}}$ & $71.64^{\mathrm{c}}$ & $73.73^{\mathrm{ab}}$ & $74.93^{\mathrm{a}}$ & $71.72^{\mathrm{c}}$ & $72.47^{\mathrm{bc}}$ & 0.61 \\
\hline $\mathrm{CP}$ & $56.67^{\mathrm{c}}$ & $57.94^{\mathrm{b}}$ & $58.35^{\mathrm{ab}}$ & $57.98^{\mathrm{b}}$ & $59.06^{\mathrm{a}}$ & $58.97^{\mathrm{a}}$ & 0.17 \\
\hline Crude lipid & $19.58^{\mathrm{e}}$ & $20.00^{\mathrm{d}}$ & $19.94^{\mathrm{d}}$ & $20.95^{\mathrm{c}}$ & $22.48^{\mathrm{a}}$ & $21.93^{\mathrm{a}}$ & 0.32 \\
\hline Crude ash & $16.30^{\mathrm{c}}$ & $16.41^{\mathrm{c}}$ & $17.36^{\mathrm{b}}$ & $16.49^{c}$ & $17.99^{\mathrm{a}}$ & $17.22^{\mathrm{b}}$ & 0.26 \\
\hline \multicolumn{8}{|c|}{ Morphological indices } \\
\hline $\mathrm{CF}^{8}$ & $1.11^{\mathrm{b}}$ & $1.73^{\mathrm{a}}$ & $1.74^{\mathrm{a}}$ & $1.77^{\mathrm{a}}$ & $1.83^{\mathrm{a}}$ & $1.85^{\mathrm{a}}$ & 0.07 \\
\hline $\mathrm{HSI}^{9}$ & $3.70^{\mathrm{d}}$ & $3.73^{\mathrm{d}}$ & $4.24^{\mathrm{c}}$ & $4.37^{\mathrm{bc}}$ & $4.60^{\mathrm{ab}}$ & $4.79^{\mathrm{a}}$ & 0.11 \\
\hline $\mathrm{VSI}^{10}$ & $11.71^{\mathrm{d}}$ & $12.27^{\mathrm{c}}$ & $13.26^{\mathrm{bc}}$ & $13.63^{\mathrm{b}}$ & $14.73^{\mathrm{a}}$ & $15.16^{\mathrm{a}}$ & 0.32 \\
\hline \multicolumn{8}{|c|}{ Serological characteristics } \\
\hline HCT & $31.73^{\mathrm{c}}$ & $31.63^{\mathrm{c}}$ & $33.87^{\mathrm{a}}$ & $32.77^{\mathrm{b}}$ & $31.27^{\mathrm{c}}$ & $33.07^{\mathrm{ab}}$ & 0.22 \\
\hline $\mathrm{Hb}$ & 4.17 & 4.00 & 4.07 & 4.07 & 4.23 & 4.00 & 0.02 \\
\hline GOT & $51.77^{\mathrm{a}}$ & $51.47^{\mathrm{ab}}$ & $50.67^{\mathrm{d}}$ & $51.17^{\mathrm{bc}}$ & $50.78^{\mathrm{cd}}$ & $51.53^{\mathrm{ab}}$ & 0.24 \\
\hline GPT & $11.47^{\mathrm{a}}$ & $11.40^{\mathrm{ab}}$ & $10.83^{\mathrm{b}}$ & $11.37^{\mathrm{ab}}$ & $11.43^{\mathrm{a}}$ & $11.27^{\mathrm{ab}}$ & 0.16 \\
\hline
\end{tabular}

Pooled SEM, pooled standard error of means: $\mathrm{SD} / \sqrt{\mathrm{n}}$; HCT, hematocrit (\%); Hb, hemoglobin $(\mathrm{g} / \mathrm{dL})$; GOT, glutamic oxaloacetic transaminase (IU/L); GPT, glutamic pyruvic transaminase (IU/L).

${ }^{1}$ Values are means from triplicate groups of fish where the values in each row with different superscripts are significantly different $(p<0.05)$.

${ }^{2}$ Satiation $(3.7 \%)=$ Total feed weight $(\mathrm{g}) \times 100 /$ total fish weight $(\mathrm{g}) .{ }^{3}$ Weight gain $(\%)=($ Final weight - initial weight $) \times 100 /$ initial weight.

${ }^{4}$ Specific growth rate $(\% / \mathrm{d})=\left(\log _{\mathrm{e}}\right.$ final wt $-\log _{\mathrm{e}}$ initial wt $) \times 100 / \mathrm{d} .{ }^{5}$ Feed efficiency $(\%)=$ Wet weight gain $(\mathrm{g}) \times 100 /$ dry feed intake $(\mathrm{g})$.

${ }^{6}$ Protein efficiency ratio $=$ Wet weight gain/protein intake. ${ }^{7}$ Survival $(\%)=$ Number of fish at end of experiment $\times 100 /$ number of fish stocked.

${ }^{8}$ Condition factor $=$ Fish weight $(\mathrm{g}) \times 100 /$ total length $\left(\mathrm{cm}^{3}\right) .{ }^{9}$ Hepatosomatic index $=$ Liver weight $(\mathrm{g}) \times 100 /$ fish weight $(\mathrm{g})$.

${ }^{10}$ Visceralsomatic index $=$ Visceral weight $\times 100 /$ fish weight $(\mathrm{g})$.

${ }^{a, b}, c$ Within a row, means without a common letters $(a, b$, and $c)$ differ $(\mathrm{p}<0.05)$.

Whole-body proximate composition of juvenile Korean rockfish reared at $16^{\circ} \mathrm{C}$ water temperature is shown in Table 1. Whole-body CP, crude lipid content of fish fed groups of $3.4 \%$ and satiation $(3.7 \% \mathrm{BW} / \mathrm{d})$ were significantly higher and fish fed group of $1.5 \% \mathrm{BW} / \mathrm{d}$ was significantly lower than those of other fish fed $(p<0.05)$ groups. There were no

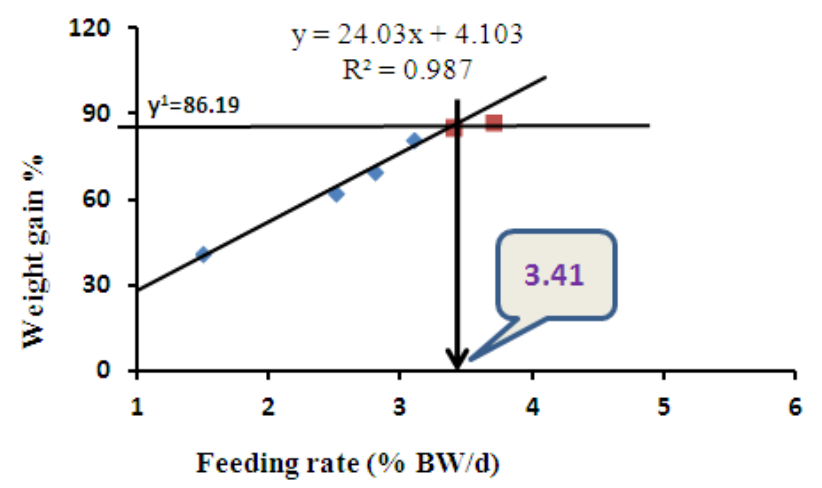

Figure 1. Broken line analysis of weight gain of $16 \mathrm{~g}$ juvenile Korean rockfish, reared at $16^{\circ} \mathrm{C}$ water temperature. clear trends with feeding rates among whole-body $\mathrm{CP}$, crude lipid, whole-body moisture and crude ash content of fish in all the treatments. Crude fiber contents of fish fed at 3.4\% and satiation $(\% \mathrm{BW} / \mathrm{d})$ groups are not different from those of fish fed at $2.5 \%, 2.8 \%$, or $3.1 \% \mathrm{BW} / \mathrm{d}$. Morphological indices such as condition factor, hepatosomatic index and visceral somatic index of fish were gradually increased as the increasing of feeding rate (Table 1). Morphological indices CF, HSI and VSI of fish fed at $3.4 \%$ and satiation (\% BW/d) groups were significantly higher than all other groups of fish fed. There were no significant differences of HSI of fish fed at $1.5 \%$ and $2.5 \%(\mathrm{p}<0.05)$ groups.

Serological characteristics of $16 \mathrm{~g}$ juvenile Korean rockfish reared at $16^{\circ} \mathrm{C}$ are depicted in Table 1 . Hematocrit of the fish fed at $2.8 \%$ and satiation $(3.7 \% \mathrm{BW} / \mathrm{d})$ were significantly higher than those of fish fed groups of $1.5 \%$, $2.5 \%, 3.1 \%$, and $3.4 \% \mathrm{BW} / \mathrm{d}(\mathrm{p}<0.05)$. There was no significant difference of hemoglobin of fish among all the feeding rate groups. Glutamic oxaloacetic transaminase and glutamic pyruvic transaminase of the fish fed at $1.5 \%$ was 
significantly higher than all other fish fed groups. There were no significant differences in these parameters among all the feeding treatments.

\section{Feeding rates for $16 \mathrm{~g}$ of juvenile Korean rockfish reared at $20^{\circ} \mathrm{C}$}

After four weeks of feeding trials at $20^{\circ} \mathrm{C}$ experiment of Korean rockfish, weight gain and specific growth rate of fish fed groups of $3.8 \%$ and satiation $(4.1 \% \mathrm{BW} / \mathrm{d})$ were significantly higher than those of fish fed groups of $1.9 \%$ and $2.9 \%(p<0.05)$ are shown in Table 2. Feed efficiency and protein efficiency ratio of fish fed at $3.5 \%$ groups were significantly higher than those of other fish fed groups $(\mathrm{p}<0.05)$. There were no significant differences of FE among the fish fed groups at $1.9 \%$ and $3.8 \% \mathrm{BW} / \mathrm{d}$ and PER among the fish fed groups at $1.9 \%, 2.9 \%$, and $3.2 \% \mathrm{BW} / \mathrm{d}$ (Table 2). Survival rate of fish fed at $1.9 \%$ and also at satiation fed $(4.1 \% \mathrm{BW} / \mathrm{d})$ were significantly lower and fish fed at $3.2 \%$ and $3.8 \%$ were significantly higher than other fish fed groups $(\mathrm{p}<0.05)$. Broken line analysis of weight gain indicated that the optimum feeding rates of juvenile Korean rockfish was $3.75 \% \mathrm{BW} / \mathrm{d}$ (Figure 2). Therefore, the results demonstrated that the optimum daily feeding rate of juvenile Korean rockfish could be greater than $3.75 \%$ but less than $4.10 \%$ at $20^{\circ} \mathrm{C}$ water temperature.

Whole-body proximate composition and morphological indices of $16 \mathrm{~g}$ juvenile Korean rockfish fed commercial diet at $20^{\circ} \mathrm{C}$ water temperature are shown in Table 2 . Whole-body CP and crude ash content of fish fed groups at $3.8 \%$ and satiation $(4.1 \% \mathrm{BW} / \mathrm{d})$ were significantly higher and fish fed group of $1.9 \%$ was significantly lower than other fish fed groups. Crude lipid of fish fed at $1.9 \% \mathrm{BW} / \mathrm{d}$ was significantly lower and fish fed at $3.8 \%$ was significantly higher than those of other fish fed groups $(p<0.05)$. There were no clear trends in whole-body moisture content of fish in all treatments. Condition factor, hepatosomatic index and visceral somatic index of fish were gradually increased as the increasing of feeding rate. Morphological indices CF, HSI and VSI of fish fed group at $1.9 \%$ were significantly lower and fish fed groups of $3.8 \%$

Table 2. Growth performance, whole-body proximate composition, morphological indices and serological characteristics of $16 \mathrm{~g}$ fish reared at $20^{\circ} \mathrm{C}$ water temperature ${ }^{1}$

\begin{tabular}{|c|c|c|c|c|c|c|c|}
\hline \multirow{2}{*}{ Item } & \multicolumn{7}{|c|}{ Diets } \\
\hline & $1.9 \%$ & $2.9 \%$ & $3.2 \%$ & $3.5 \%$ & $3.8 \%$ & $\mathrm{SA}^{2}$ & Pooled SEM \\
\hline \multicolumn{8}{|c|}{ Growth performance } \\
\hline $\mathrm{WG}^{3}$ & $51.94^{\mathrm{f}}$ & $75.16^{\mathrm{e}}$ & $80.12^{\mathrm{d}}$ & $88.23^{\mathrm{c}}$ & $95.45^{\mathrm{b}}$ & $96.75^{\mathrm{a}}$ & 3.93 \\
\hline $\mathrm{SGR}^{4}$ & $1.75^{\mathrm{d}}$ & $2.34^{\mathrm{c}}$ & $2.47^{\mathrm{bc}}$ & $2.63^{\mathrm{ab}}$ & $2.80^{\mathrm{a}}$ & $2.81^{\mathrm{a}}$ & 0.09 \\
\hline $\mathrm{FE}^{5}$ & $87.63^{\mathrm{d}}$ & $88.49^{\mathrm{c}}$ & $89.25^{\mathrm{b}}$ & $99.53^{\mathrm{a}}$ & $87.53^{\mathrm{d}}$ & $79.55^{\mathrm{e}}$ & 3.56 \\
\hline $\mathrm{PER}^{6}$ & $1.86^{\mathrm{b}}$ & $1.87^{\mathrm{b}}$ & $1.87^{\mathrm{b}}$ & $2.11^{\mathrm{a}}$ & $1.79^{\mathrm{bc}}$ & $1.69^{c}$ & 0.07 \\
\hline Survival $^{7}$ & $98.24^{\mathrm{c}}$ & $99.56^{\mathrm{b}}$ & $100^{\mathrm{a}}$ & $99.54^{\mathrm{b}}$ & $100^{\mathrm{a}}$ & $98.34^{\mathrm{c}}$ & 0.06 \\
\hline \multicolumn{8}{|c|}{ Whole-body proximate composition } \\
\hline Moisture & $73.46^{\mathrm{bc}}$ & $72.63^{d}$ & $74.36^{\mathrm{d}}$ & $75.38^{\mathrm{b}}$ & $72.27^{\mathrm{d}}$ & $73.74^{\mathrm{bc}}$ & 0.51 \\
\hline $\mathrm{CP}$ & $55.46^{\mathrm{d}}$ & $57.54^{\mathrm{ab}}$ & $56.56^{\mathrm{b}}$ & $58.58^{\mathrm{b}}$ & $60.12^{\mathrm{a}}$ & $59.95^{\mathrm{a}}$ & 0.16 \\
\hline Crude lipid & $20.56^{\mathrm{d}}$ & $21.00^{\mathrm{ab}}$ & $20.95^{\mathrm{bc}}$ & $21.68^{\mathrm{b}}$ & $22.54^{\mathrm{a}}$ & $21.93^{\mathrm{b}}$ & 0.41 \\
\hline Crude ash & $17.03^{\mathrm{b}}$ & $17.15^{\mathrm{b}}$ & $18.64^{\mathrm{a}}$ & $17.85^{\mathrm{ab}}$ & $18.66^{\mathrm{a}}$ & $18.35^{\mathrm{a}}$ & 0.34 \\
\hline \multicolumn{8}{|c|}{ Morphological indices } \\
\hline $\mathrm{CF}^{8}$ & $1.12^{\mathrm{b}}$ & $1.77^{\mathrm{a}}$ & $1.79^{\mathrm{a}}$ & $1.82^{\mathrm{a}}$ & $1.88^{\mathrm{a}}$ & $1.97^{\mathrm{a}}$ & 0.07 \\
\hline $\mathrm{HSI}^{9}$ & $3.87^{\mathrm{c}}$ & $3.87^{\mathrm{c}}$ & $4.37^{\mathrm{b}}$ & $4.67^{\mathrm{ab}}$ & $4.90^{\mathrm{a}}$ & $4.99^{\mathrm{a}}$ & 0.12 \\
\hline $\mathrm{VSI}^{10}$ & $12.01^{\mathrm{d}}$ & $12.61^{\mathrm{c}}$ & $13.57^{\mathrm{bc}}$ & $14.56^{\mathrm{b}}$ & $15.52^{\mathrm{a}}$ & $15.92^{\mathrm{a}}$ & 0.37 \\
\hline \multicolumn{8}{|c|}{ Serological characteristics } \\
\hline $\mathrm{HCT}$ & $31.0^{\mathrm{ab}}$ & $32.7^{\mathrm{a}}$ & $30.1^{\mathrm{b}}$ & $32.3^{\mathrm{a}}$ & $31.9^{\mathrm{bc}}$ & $32.6^{\mathrm{a}}$ & 0.24 \\
\hline $\mathrm{Hb}$ & 4.17 & 4.10 & 4.17 & 3.97 & 4.23 & 4.07 & 0.09 \\
\hline GOT & $50.40^{\mathrm{a}}$ & $49.13^{\mathrm{b}}$ & $50.11^{\mathrm{a}}$ & $49.40^{\mathrm{b}}$ & $50.31^{\mathrm{a}}$ & $50.32^{\mathrm{a}}$ & 0.36 \\
\hline GPT & $10.43^{\mathrm{a}}$ & $9.27^{\mathrm{bc}}$ & $10.30^{\mathrm{a}}$ & $9.71^{\mathrm{b}}$ & $9.13^{\mathrm{c}}$ & $10.32^{\mathrm{a}}$ & 0.06 \\
\hline
\end{tabular}

Pooled SEM, pooled standard error of means: SD/ $\sqrt{\mathrm{n}}$; HCT, hematocrit $(\%)$; Hb, hemoglobin (g/dL); GOT, glutamic oxaloacetic transaminase (IU/L); GPT, glutamic pyruvic transaminase (IU/L).

${ }^{1}$ Values are means from triplicate groups of fish where the values in each row with different superscripts are significantly different (p<0.05).

${ }^{2}$ Satiation $(4.1 \%)=$ Total feed weight $(\mathrm{g}) \times 100 /$ total fish weight $(\mathrm{g}) .{ }^{3}$ Weight gain $(\%)=($ Final weight - initial weight $) \times 100 /$ initial weight .

${ }^{4}$ Specific growth rate $(\% / \mathrm{d})=\left(\log _{\mathrm{e}}\right.$ final wt $-\log _{\mathrm{e}}$ initial $\left.\mathrm{wt}\right) \times 100 / \mathrm{d} .{ }^{5}$ Feed efficiency $(\%)=$ Wet weight gain $(\mathrm{g}) \times 100 / \mathrm{dry}$ feed intake $(\mathrm{g})$.

${ }^{6}$ Protein efficiency ratio $=$ Wet weight gain/protein intake. ${ }^{7}$ Survival $(\%)=$ Number of fish at end of experiment $\times 100 /$ Number of fish stocked.

${ }^{8}$ Condition factor $=$ Fish weight $(\mathrm{g}) \times 100 /$ total length $\left(\mathrm{cm}^{3}\right) .{ }^{9}$ Hepatosomatic index $=$ Liver weight $(\mathrm{g}) \times 100 /$ fish weight $(\mathrm{g})$.

${ }^{10}$ Visceralsomatic index $=$ Visceral weight $\times 100 /$ fish weight $(\mathrm{g})$.

a,b,c Within a row, means without a common letters $(a, b$, and c) differ $(\mathrm{p}<0.05)$. 


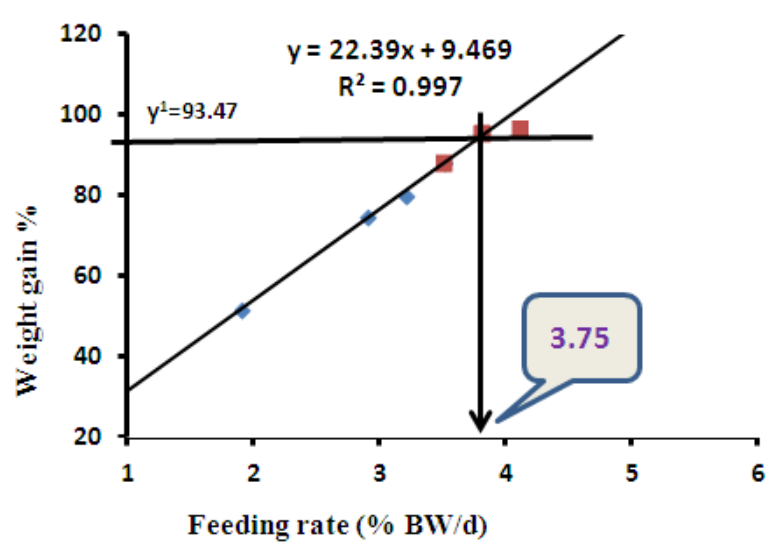

Figure 2. Broken line analysis of weight gain of $16 \mathrm{~g}$ juvenile Korean rockfish, reared at $20^{\circ} \mathrm{C}$ water temperature.

and satiation $(4.1 \% \mathrm{BW} / \mathrm{d})$ were significantly higher than other groups of fish fed. There were no significant differences of HSI of fish fed groups of $1.9 \%$ and $2.9 \%$ (BW/d).

Serological characteristics of $16 \mathrm{~g}$ juvenile Korean rockfish reared at $20^{\circ} \mathrm{C}$ are depicted in Table 2. Hematocrit of the fish fed at $3.2 \%$ (BW/d) was significantly lower than other fish fed groups. There were no significant differences in hematocrit of fish in the fish fed groups at $2.9 \%, 3.5 \%$ and satiation $(4.1 \% \mathrm{BW} / \mathrm{d})$ and it was significantly higher than other fish fed groups. No significant differences were found in hemoglobin of fish in all treatments. Serum GOT and GPT amount of the fish fed at 1.9\%, 3.3\% and satiation $(4.1 \% \mathrm{BW} / \mathrm{d})$ were significantly higher in juvenile Korean rockfish.

\section{Feeding rates for $16 \mathrm{~g}$ of juvenile Korean rockfish reared at $24^{\circ} \mathrm{C}$}

At the end of 4 wk of feeding trials in $24^{\circ} \mathrm{C}$ experiment of juvenile Korean rockfish, weight gain and specific growth rate of fish fed at 3.3\%, 3.6\% and satiation $(3.9 \%$ $\mathrm{BW} / \mathrm{d})$ were no significant differences and these were significantly higher than those of fish fed of $1.7 \%, 2.7 \%$, and $3.0 \%$ fish fed groups $(\mathrm{p}<0.05)$ are shown in Table 3. There were no significant differences of WG and SGR among fish fed at $2.7 \%$ and $3.0 \% \mathrm{BW} / \mathrm{d}$. Weight gain,

Table 3. Growth performance, whole-body proximate composition, morphological indices and serological characteristics of $16 \mathrm{~g}$ fish reared at $24^{\circ} \mathrm{C}$ water temperature ${ }^{1}$

\begin{tabular}{|c|c|c|c|c|c|c|c|}
\hline \multirow{2}{*}{ Item } & \multicolumn{7}{|c|}{ Diets } \\
\hline & $1.7 \%$ & $2.7 \%$ & $3.0 \%$ & $3.3 \%$ & $3.6 \%$ & $\mathrm{SA}^{2}$ & Pooled SEM \\
\hline \multicolumn{8}{|c|}{ Growth performance } \\
\hline $\mathrm{WG}^{3}$ & $49.34^{\mathrm{c}}$ & $74.50^{\mathrm{b}}$ & $77.41^{\mathrm{b}}$ & $83.66^{\mathrm{a}}$ & $86.36^{\mathrm{a}}$ & $87.17^{\mathrm{a}}$ & 3.34 \\
\hline $\mathrm{SGR}^{4}$ & $1.67^{\mathrm{c}}$ & $2.32^{\mathrm{b}}$ & $2.39^{\mathrm{b}}$ & $2.53^{\mathrm{a}}$ & $2.59^{\mathrm{a}}$ & $2.61^{\mathrm{a}}$ & 0.08 \\
\hline $\mathrm{FE}^{5}$ & $80.21^{\mathrm{c}}$ & $81.27^{\mathrm{c}}$ & $84.62^{\mathrm{b}}$ & $93.12^{\mathrm{a}}$ & $85.66^{\mathrm{b}}$ & $80.70^{\mathrm{c}}$ & 1.51 \\
\hline $\mathrm{PER}^{6}$ & $1.70^{\mathrm{ab}}$ & $1.72^{\mathrm{ab}}$ & $1.79^{\mathrm{ab}}$ & $1.97^{\mathrm{a}}$ & $1.69^{\mathrm{b}}$ & $1.56^{\mathrm{b}}$ & 0.03 \\
\hline Survival $^{7}$ & 95.21 & 95.61 & 96.62 & 95.81 & 96.82 & 95.81 & 0.15 \\
\hline \multicolumn{8}{|c|}{ Whole-body proximate composition } \\
\hline Moisture & $72.31^{\mathrm{b}}$ & $72.41^{\mathrm{b}}$ & $73.56^{\mathrm{ab}}$ & $74.83^{\mathrm{a}}$ & $71.73^{\mathrm{c}}$ & $72.76^{\mathrm{b}}$ & 0.35 \\
\hline $\mathrm{CP}$ & $54.36^{\mathrm{d}}$ & $56.55^{\mathrm{bc}}$ & $55.97^{\mathrm{c}}$ & $57.76^{\mathrm{b}}$ & $58.42^{\mathrm{a}}$ & $58.59^{\mathrm{a}}$ & 0.14 \\
\hline Crude lipid & $20.12^{\mathrm{c}}$ & $20.59^{\mathrm{ab}}$ & $21.42^{\mathrm{a}}$ & $20.75^{\mathrm{b}}$ & $21.15^{\mathrm{a}}$ & $20.87^{\mathrm{b}}$ & 0.36 \\
\hline Crude ash & $16.02^{\mathrm{b}}$ & $16.15^{\mathrm{b}}$ & $17.53^{\mathrm{a}}$ & $16.84^{\mathrm{ab}}$ & $17.54^{\mathrm{a}}$ & $17.18^{\mathrm{a}}$ & 0.31 \\
\hline \multicolumn{8}{|c|}{ Morphological indices } \\
\hline $\mathrm{CF}^{8}$ & $1.07^{\mathrm{b}}$ & $1.76^{\mathrm{a}}$ & $1.78^{\mathrm{a}}$ & $1.77^{\mathrm{a}}$ & $1.83^{\mathrm{a}}$ & $1.86^{\mathrm{a}}$ & 0.07 \\
\hline $\mathrm{HSI}^{9}$ & $3.73^{\mathrm{d}}$ & $3.74^{\mathrm{d}}$ & $4.24^{\mathrm{c}}$ & $4.46^{\mathrm{bc}}$ & $4.72^{\mathrm{ab}}$ & $4.84^{\mathrm{a}}$ & 0.11 \\
\hline $\mathrm{VSI}^{10}$ & $11.8^{\mathrm{b}}$ & $12.3^{\mathrm{b}}$ & $13.2^{\mathrm{ab}}$ & $13.9^{\mathrm{ab}}$ & $15.1^{\mathrm{a}}$ & $15.3^{\mathrm{a}}$ & 0.34 \\
\hline \multicolumn{8}{|c|}{ Serological characteristics } \\
\hline $\mathrm{HCT}$ & $29.47^{\mathrm{b}}$ & $31.37^{\mathrm{a}}$ & $31.20^{\mathrm{a}}$ & $30.90^{\mathrm{ab}}$ & $31.70^{\mathrm{a}}$ & $30.10^{\mathrm{ab}}$ & 0.20 \\
\hline $\mathrm{Hb}$ & 4.40 & 3.83 & 4.00 & 3.93 & 4.03 & 3.90 & 0.02 \\
\hline GOT & $52.43^{\mathrm{ab}}$ & $51.83^{\mathrm{cd}}$ & $52.70^{\mathrm{a}}$ & $51.53^{\mathrm{d}}$ & $52.87^{\mathrm{a}}$ & $52.23^{\mathrm{bc}}$ & 0.15 \\
\hline GPT & $12.27^{\mathrm{a}}$ & $11.67^{\mathrm{b}}$ & $12.17^{\mathrm{ab}}$ & $11.93^{\mathrm{ab}}$ & $12.33^{\mathrm{a}}$ & $12.11^{\mathrm{ab}}$ & 0.07 \\
\hline
\end{tabular}

Pooled SEM, pooled standard error of means: SD/ $\sqrt{\mathrm{n}}$; HCT, hematocrit (\%); Hb, hemoglobin (g/dL); GOT, glutamic oxaloacetic transaminase (IU/L); GPT, glutamic pyruvic transaminase (IU/L).

${ }^{1}$ Values are means from triplicate groups of fish where the values in each row with different superscripts are significantly different ( $\mathrm{p}<0.05$ ).

${ }^{2}$ Satiation $(3.9 \%)=$ Total feed weight $(\mathrm{g}) \times 100 /$ total fish weight $(\mathrm{g}) .{ }^{3}$ Weight gain $(\%)=($ Final weight - initial weight $) \times 100 /$ initial weight

${ }^{4}$ Specific growth rate $(\% / \mathrm{d})=\left(\log _{\mathrm{e}}\right.$ final wt $-\log _{\mathrm{e}}$ initial $\left.\mathrm{wt}\right) \times 100 / \mathrm{d} .{ }^{5}$ Feed efficiency $(\%)=$ Wet weight gain $(\mathrm{g}) \times 100 / \mathrm{dry}$ feed intake $(\mathrm{g})$.

${ }^{6}$ Protein efficiency ratio $=$ Wet weight gain/protein intake. ${ }^{7}$ Survival $(\%)=$ Number of fish at end of experiment $\times 00 /$ number of fish stocked.

${ }^{8}$ Condition factor $=$ Fish weight $(\mathrm{g}) \times 100 /$ total length $\left(\mathrm{cm}^{3}\right) .{ }^{9}$ Hepatosomatic index $=$ Liver weight $(\mathrm{g}) \times 100 /$ fish weight $(\mathrm{g})$.

${ }^{10}$ Visceralsomatic index $=$ Visceral weight $\times 100 /$ fish weight $(\mathrm{g})$.

a,b,c Within a row, means without a common letters $(a, b$, and c) differ $(\mathrm{p}<0.05)$. 
specific growth rate and feed efficiency of fish fed group of $1.7 \% \mathrm{BW} / \mathrm{d}$ was significantly lower than those of other fish fed groups. Protein efficiency ratio and FE of fish fed group of $3.3 \% \mathrm{BW} / \mathrm{d}$ were significantly higher than those of other fish fed groups. On the other hand, there was no significant difference of FE at the fish fed groups of $1.7 \%$ and $2.7 \%$ and at satiation $(4.10 \% \mathrm{BW} / \mathrm{d})$. No significant differences were found in survival rate among all the fish fed groups. Broken line analysis of weight gain indicated that the optimum feeding rates of this fish was $3.34 \%$ BW/d (Figure $3)$. Therefore, the results revealed that the optimum daily feeding rate of juvenile Korean rockfish could be greater than $3.34 \%$ but less than $3.90 \%$ at $24^{\circ} \mathrm{C}$ water temperature.

Table 3 shows the whole-body proximate composition of $16 \mathrm{~g}$ juvenile Korean rockfish reared at $24^{\circ} \mathrm{C}$ water temperature. Whole-body CP was increased with increasing of feeding rate though there was slight drop in the fish fed at $3.3 \% \mathrm{BW} / \mathrm{d}$. There were no significant differences between the fish fed group of $3.6 \%$ and satiation $(4.1 \%$ $\mathrm{BW} / \mathrm{d})$ and it was significantly higher than those of other fish fed groups. Crude lipid content of fish fed group of $1.7 \%$ was significantly lower than other fish fed groups. On the other hand, no significant differences were found in whole body crude ash content between the fish fed groups of $3.6 \%$ and satiation $(4.1 \% \mathrm{BW} / \mathrm{d})$. There were no clear trends in whole-body moisture and crude lipid and crude ash content of fish in all feeding rates at $24^{\circ} \mathrm{C}$ water temperature.

Morphological indices and serological characteristics of $16 \mathrm{~g}$ juvenile Korean rockfish reared at $24^{\circ} \mathrm{C}$ are shown in Table 3. Condition factor, HSI and VSI of fish were gradually increased as the increasing of feeding rate. Hepatosomatic index of fish fed group of satiation $(3.9 \%$ $\mathrm{BW} / \mathrm{d}$ ) and VSI of fish fed groups of $3.6 \%$ and satiation fish groups were significantly higher than other groups of fish fed. No significant differences were found in HSI and VSI of fish fed groups of $1.7 \%$ and $2.7 \%(\mathrm{BW} / \mathrm{d})$ and it was significantly lower than other fish fed groups. On the other hand, VSI of fish fed of $3.0 \%$ and $3.3 \%$ groups $(p<0.05)$ did

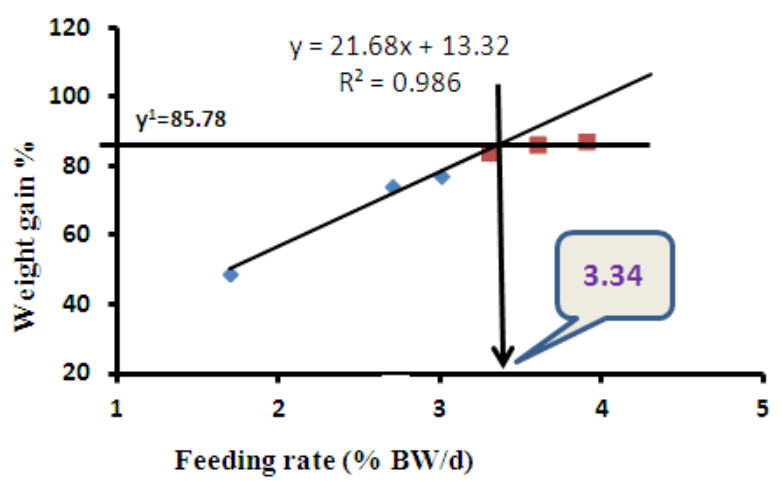

Figure 3. Broken line analysis of weight gain of $16 \mathrm{~g}$ juvenile Korean rockfish, reared at $24^{\circ} \mathrm{C}$ water temperature. not show any significant difference. Hematocrit of fish fed group at $1.7 \%$ was significantly lower and fish fed groups of $2.7 \%, 3.0 \%$, and $3.6 \%(\mathrm{BW} / \mathrm{d})$ were significantly higher than those of other fish fed groups. There were no significant differences of hemoglobin contents of all the fish fed groups. Serum GOT of the fish fed groups of $3.0 \%$ and $3.6 \%$ and GPT of the fish fed groups of $1.7 \%$ and $3.6 \%$ (BW/d) were significantly higher and GPT of fish fed group of $2.7 \%$ and GOT of fish fed group of $3.3 \%$ were significantly lower than those all other fish fed groups.

\section{DISCUSSION}

Three feeding trial experiments (at $16^{\circ} \mathrm{C}, 20^{\circ} \mathrm{C}$, and $24^{\circ} \mathrm{C}$ ) were conducted to determine the effect of feeding rate and water temperature on biological performance, morphological indices, whole-body proximate composition and serological characteristics of juvenile Korean rockfish. Weight gain and SGR were increased with increasing of feeding rate up to satiation level, although the improvement rates were different in the feeding trials. In the present study feed efficiency and protein efficiency ratio were also increased with the increasing of feeding rate up to the optimum level. On the other hand, feed utilization was leveled out after a certain feeding rate in all feeding trials. Feed efficiency and protein efficiency ratio has been increased gradually but there was a drop after the peak in all experiment. In this study beyond the peak, fish could not efficiently utilize the additional feeds, leading to the drop in feed efficiency and protein efficiency ratio (Tables 1 to 3 ) which also has got the same pattern of $5 \mathrm{~g}$ of juvenile olive flounder fish described by Okorie et al. (2013).

Fish appear to continuously increase in weight with the increasing feeding rate up to satiation at $16^{\circ} \mathrm{C}, 20^{\circ} \mathrm{C}$, and $24^{\circ} \mathrm{C}$ experiments although this increase may not be corresponded always with the incremental feed consumption. Weight gain also continuously increases with increasing of feeding rate has been recorded in white sturgeon, Acipenser transmontanus (Deng et al., 2003); European sea bass, Dicentrarchus labrax (Eroldogan et al., 2004); olive flounder, Paralichthys olivaceus (Cho et al., 2007), blackspot seabream, Pagellus bogaraveo (Ozorio et al., 2009) and juvenile Korean rockfish, Sebastes schlegeli (Mizanur et al., 2013).

Fish tend to optimize their digestion to extract nutrients more efficiently at lower feeding rates (Van Ham et al., 2003). Present experiments indicated that optimum feeding rates in $16 \mathrm{~g}$ juvenile Korean rockfish were below the satiation level of feeding rates. Many researchers found that optimum feeding rates were below the satiation level in European sea bass (Eroldogan et al., 2004) and juvenile olive flounder (Kim et al., 2007) and juvenile Korean rockfish (Mizanur et al., 2013). In the present studies the 
optimum range of feeding rates at $16^{\circ} \mathrm{C}, 20^{\circ} \mathrm{C}$, and $24^{\circ} \mathrm{C}$ were $3.1 \%$ to $3.41 \% \mathrm{BW} / \mathrm{d}, 3.5 \%$ to $3.75 \% \mathrm{BW} / \mathrm{d}$, and $3.0 \%$ to $3.34 \% \mathrm{BW} / \mathrm{d}$ respectively. Among the experiments of $16^{\circ} \mathrm{C}, 20^{\circ} \mathrm{C}$, and $24^{\circ} \mathrm{C}$ growth rate of $16 \mathrm{~g}$ size Korean rockfish was relatively higher at $20^{\circ} \mathrm{C}$. Azaza et al. (2008) found that lower and upper of the optimum water temperature significantly affected the growth, survival and feed efficiency of Nile tilapia, Oreochromis niloticus.

Hepatosomatic index and condition factor may provide the information on growth, physical condition, energy reserves and ability of fish to tolerate the environmental stresses. In a poor, stressful and unfavorable environment, fish usually have a smaller liver which means less energy reserved in the liver. In the present studies HSI, VSI, and $\mathrm{CF}$ were increased with the increasing of feeding rate in all the experiments. The researchers got the same results in the previous study on feeding rates in $5 \mathrm{~g}$ of juvenile Korean rockfish (Mizanur et al., 2013). Hepatosomatic index is a good compensatory growth index in olive flounder (Cho, 2005), and Juvenile black rockfish, Sebastes schlegeli (Oh et al., 2008). Hepatosomatic index and condition factor of largescale tonguesole, Cynoglossus arel has shown the seasonal trend (to increase and decrease) round the year (Ghaffari et al., 2011). In the present study HSI, VSI, and $\mathrm{CF}$ shows better growth at $20^{\circ} \mathrm{C}$ than those at $16^{\circ} \mathrm{C}$ and $24^{\circ} \mathrm{C}$ (Tables 1 to 3 ).

The plasma transaminase enzymes aspartate aminotransferase (AST) and alanine aminotransferase (ALT) are found not only in liver but also in red blood cells, heart cells, muscle tissue, pancreas and kidneys (Huang et al., 2006). Those of AST and ALT formerly are called GOT and GPT respectively. Serum GPT and GOT ratio provide useful information regarding the extent and causes of liver disease of olive flounder (Qiao et al., 2012). In the present study serological characteristics of juvenile Korean rockfish seem not to be affected by different feeding rates. There were no clear trends in hemoglobin, hematocrit, serum GOT and GPT of juvenile Korean rockfish fed commercial diet at different feeding rates at $16^{\circ} \mathrm{C}, 20^{\circ} \mathrm{C}$, and $24^{\circ} \mathrm{C}$. Serum GPT and GOT level of $20 \mathrm{~g}$ olive flounder fish did not affected by different dietary feeding treatments (Okorie et al., 2013). Cho et al. (2006) did not observe any significant differences in serum glucose, total protein or GPT of olive flounder fed extruded pellets at different feeding ratios. Furthermore, serum total protein, glucose and GPT of flounder were not significantly affected by feeding ratio while GOT fluctuated as reported by Cho et al. (2007). The present study has been indicated that serum GPT and GOT in the group of fish fed at lowest feeding rates $1.5 \%$ of $16^{\circ} \mathrm{C}, 1.9 \%$ of $20^{\circ} \mathrm{C}$ and $1.7 \%$ of $24^{\circ} \mathrm{C}$, were a slightly higher than other fish fed groups (Tables 1 to 3 ) because fish might be always in stress of underfeeding.

Water temperature has substantial effect on fish metabolism. In response to decreasing of water temperature the enzyme activity of tissues have been increased (Hochachka and Somero, 1984). Velmurugan et al. (2007) have investigated that histopathological and tissue enzyme changes of $C$. gariepinus exposed to nitrite when water temperatures changes from $27^{\circ} \mathrm{C}$ to $35^{\circ} \mathrm{C}$. In a stressful and unfavorable environmental condition GPT and GOT may increase in blood serum. In the present study serum GPT and GOT level were affected by different water temperature. Serum GPT and GOT amount in different fish fed at $20^{\circ} \mathrm{C}$ are comparatively lower than those of fish fed at $16^{\circ} \mathrm{C}$ and $24^{\circ} \mathrm{C}$ experiments (Tables 1 to 3 ). These results indicated that $20^{\circ} \mathrm{C}$ may be a favorable water temperature for better growth of $16 \mathrm{~g}$ juvenile Korean rockfish.

Whole-body proximate compositions of Korean rockfish were also determined to know the optimum temperature and feeding rates. Lipid appeared to accumulate with the increasing of feeding rate at $16^{\circ} \mathrm{C}$ although the trend was not very obvious amongst the fed fish groups in all trials. Normally lipid levels of fish increase with the increased feeding rate while moisture and ash levels decrease (Abdelghany and Ahmad, 2002). Whole-body proximate composition seems not to be a perfect parameter to determine the optimum feeding rate and optimum water temperature. Wang et al. (2007) reported that an increased in moisture and ash contents with corresponding decreased in lipid content of cuneate drum, Nibea miichthioides with feeding rate, but in our study such a relationship did not observe in juvenile Korean rockfish. There were no significant differences in whole-body proximate composition of olive flounder fed at different feeding rates except for the lipid content, which was significantly lower only in fish fed at the lowest feeding rate (Kim et al., 2007).

Survival rates were not affected significantly by different feeding rates but survival rate at lowest feeding rates of $16^{\circ} \mathrm{C}$ and $20^{\circ} \mathrm{C}$ were significantly lower at all the experiments. On the other hand survival rates might be affected by different water temperature because the survival rate at $20^{\circ} \mathrm{C}$ water temperature was significantly higher than the experiments of $16^{\circ} \mathrm{C}$ and $24^{\circ} \mathrm{C}$ (Tables 1 to 3). Azaza et al. (2008) described that the survival rate of Nile tilapia, Oreochromis niloticus was significantly lower when it was reared at lower and upper level of its optimum water temperature.

ANOVA indicated that optimum FE and PER could be achieved at the feeding rate below satiation level. In the present study indicated that weight gain and specific growth rate from those fish of satiation and $3.4 \%$ groups at $16^{\circ} \mathrm{C}$, fish from satiation and $3.8 \%$ groups at $20^{\circ} \mathrm{C}$ and fish from satiation and $3.6 \%$ groups at $24^{\circ} \mathrm{C}$ were significantly higher than other fish fed groups. 


\section{CONCLUSION}

In the conclusion, these studies demonstrate that growth performance of $16 \mathrm{~g}$ of juvenile Korean rockfish was affected by feeding rates and water temperatures. Growth performance appeared to be a more reliable parameter than whole-body proximate composition, biological indices and serological characteristics to determine the optimum feeding rates in juvenile Korean rockfish. Hepatosomatic index, condition factor and visceral somatic index have been increased with the increasing of feeding rates at all experiments. Furthermore, serological characteristics of juvenile Korean rockfish seem not to be affected by different feeding rates but affected by water temperatures. According to broken line regression analysis of weight gain the optimum feeding rates of juvenile Korean rockfish was $3.41 \%(\mathrm{BW} / \mathrm{d})$ at $16^{\circ} \mathrm{C}$ (Figure 1), 3.75\% (BW/d) at $20^{\circ} \mathrm{C}$ (Figure 2) and $3.34 \%\left(\mathrm{BW} / \mathrm{d}\right.$ ) at $24^{\circ} \mathrm{C}$ (Figure 3). Therefore, these results indicate that the optimum feeding rates could be $>3.1 \%$ but $<3.41 \%$ at $16^{\circ} \mathrm{C},>3.5 \%$ but $<3.75 \%$ at $20^{\circ} \mathrm{C}$ and $>3.0 \%$ but $<3.34 \%$ at $24^{\circ} \mathrm{C}$. As we expected results suggest that fish performed better at $20^{\circ} \mathrm{C}$ than $16^{\circ} \mathrm{C}$ or $24^{\circ} \mathrm{C}$ water temperature and the optimum feeding rate could be $3.1 \%$ to $3.7 \% \mathrm{BW} / \mathrm{d}$ in $16 \mathrm{~g}$ of juvenile Korean rockfish.

\section{ACKNOWLEDGMENTS}

The authors wish to thanks for the financial support from the National Fisheries Research and Development Institute (RP-2013-AQ-223), Republic of Korea. We would like to express our appreciation to all the students and staffs of the Feeds and Foods Nutrition Research Center (FFNRC) at Pukyong National University who collaborated in different ways while we were conducting our research.

\section{REFERENCES}

Abdelghany, A. E. and M. H. Ahmad. 2002. Effects of feeding rates on growth and production of Nile tilapia (Oreochromis niloticus), common carp (Cyprinus carpio) and silver carp (Hypophthalmichthys molitrix) polycultured in fertilized ponds. Aquac. Res. 33:415-423.

AOAC. 1995. Official Methods of Analysis. 16th edn. Association of Official Analytical Chemists, Arlington, Virginia.

Azaza, M. S., M. S. Dhraief, and M. M. Kraiem. 2008. Effects of water temperature on growth and sex ratio of juvenile Nile tilapia Oreochromis niloticus (Linnaeus) reared in geothermal waters in southern Tunisia. J. Therm. Biol. 33:98-105.

Bai, S. C. and O. E. Okorie. 2009. Marine fish. Korean Rockfish (Sebastes schlegeli) Production in Korea. Global Aquaculture Advocate. pp. 41-42.

Bai, S. C., S. M. Choi, K. W. Kim, and X. J. Wang. 2001. Apparent protein and phosphorus digestibilities of five different dietary protein sources in Korean rockfish, Sebastes schlegeli (Hilgendorf). Aquac. Res. 32:99-105.
Bailey, J. and A. Alanara. 2006. Effect of feed portion size on growth of rainbow trout, Oncorhynchus mykiss (Walbaum) reared at different temperatures. Aquaculture 253:728-730.

Brown, B. A. 1980. Routine hematology procedures. In: Hematology, Principles and Procedures (Ed. Lea and Febiger). Lea and Febiger Co. Philadelphia, PA. pp. 71-112.

Bjornsson, B., A. Steinarsson, and T. Arnason. 2007. Growth model for Atlantic cod (Gadus morhua): Effects of temperature and body weight on growth rate. Aquaculture 27:216-226.

Cho, S. H., Y. S. Lim, J. H. Lee, and S. Park. 2003. Effect of feeding rate and feeding frequency on survival, growth, and body composition of Ayu post-larvae Plecoglossus altivelis. J. World Aquac. Soc. 34:85-91.

Cho, S. H. 2005. Compensatory growth of juvenile flounder Paralichthys olivaceous L. and changes in biochemical composition and body condition indices during starvation and after refeeding in the winter season. J. World Aquac. Soc. 36:508-514.

Cho, S. H., S. M. Lee, B. H. Park, and S. Lee. 2006. Effect of feeding ratio on growth and body composition of juvenile olive flounder Paralichthys olivaceus fed extruded pellets during the summer season. Aquaculture 251:78-84.

Cho, S. H., S. M. Lee, B. H. Park, S. C. Ji, C. Y. Choi, J. H. Lee, Y. C. Kim, J. H. Lee, and S. Y. Oh. 2007. Effect of daily feeding ratio on growth and body composition of subadult olive flounder, Paralichthys olivaceus, fed an extruded diet during the summer season. J. World Aquac. Soc. 38:68-73.

Deng, D. F., S. Koshio, S. Yokoyama, S. C. Bai, Q. Shao, Y. Cui, and S. S. O. Hung. 2003. Effects of feeding rate on growth performance of white sturgeon (Acipenser transmontanus) larvae. Aquaculture 217:589-598.

Dwyer, K. S., J. A. Brown, C. Parrish, and S. P. Lall. 2002. Feeding frequency affects food consumption, feeding pattern and growth of juvenile yellowtail flounder (Limanda ferruginea). Aquaculture 213:279-292.

Eroldogan, O. T., M. Kumlu, and M. Aktas. 2004. Optimum feeding rates for European seabass Dicentrarchus labrax L. reared in seawater and freshwater. Aquaculture 231:501-515.

Gardeur, J. N., N. Mathis, A. Kobilinsky, and J. Brun-Bellut. 2007. Simultaneous effects of nutritional and environmental factors on growth and flesh quality of Perca fluviatilis using a fractional factorial design study. Aquaculture 273:50-63.

Ghaffari, H., A. A. Ardalan, H. H. Sahafi, M. M. Babaei, and R. Abdollahi. 2011. Annual changes in gonadosomatic index (GSI), hepatosomatic index (HSI) and condition factor (K) of Largescale tonguesole Cynoglossus arel in the coastal waters of Bandar Abbas, Persian Gulf. Aus. J. Basic and Appl. Sci. 5:1640-1646.

Hochachka, P. W. and G. N. Somero. 1984. Biochemical adaptation. Princeton University Press, Princeton, New Jersey. p. 525.

Huang, X-J., Y-K. Choi, H. S. Im, O. Yarimaga, E. Yoon, and H-S. Kim. 2006. Aspartate aminotransferase (AST/GOT) and alanine aminotransferase (ALT/GPT) detection techniques. Sensors 6:756-782.

Hung, S. S. O., P. B. Lutes, A. A. Shqueir, and F. S. Conte. 1993. Effect of feeding rate and water temperature on growth of juvenile white sturgeon (Acipenser transmontanus). 
Aquaculture 115:297-303.

Kestemont, P. and E. Barras. 2001. Environmental factors and feed intake: mechanisms and interactions. In: Food Intake in Fish (Ed. D. Houlihan, T. Boujard, and M. Jobling). Blackwell Scientific Publications Ltds, Oxford. pp. 131-156.

Kim, K. D., Y. J. Kang, K. W. Kim, and K. M. Kim. 2007. Effects of feeding rate on growth and body composition of juvenile flounder. J. World Aquac. Soc. 38:169-173.

Mizanur, R. M., G. Park, H. H. Yun, S. Lee, S. Choi, and S. C. Bai. 2013. The effects of feeding rates in juvenile Korean rockfish (Sebastes schlegeli) reared at $17^{\circ} \mathrm{C}$ and $20^{\circ} \mathrm{C}$ water temperatures. Aquacult. Int. (In press).

Molnar, T., A. Szabo, G. Szabo, C. Szabo, and C. Hancz. 2006. Effect of different dietary fat content and fat type on the growth and body composition of intensively reared pikeperch Sander lucioperca (L.). Aquacult. Nutr. 12:173-182.

Nyina-Wamwiza, L., X. L. Xu, G. Blanchard, and P. Kestemont. 2005. Effect of dietary protein, lipid and carbohydrate ratio on growth, feed efficiency and body composition of pikeperch Sander lucioperca fingerlings. Aquac. Res. 36:486-492.

Oh, S. Y., C. H. Noh, R. H. Kang, C. K. Kim, S. H. Cho, and J. Y. Jo. 2008. Compensatory growth and body composition of juvenile black rockfish Sebastes schlegeli following feed deprivation. Fish. Sci. 74:846-852.

Okorie, O. E., J. Y. Bae, K. W. Kim, M. H. Son, J. W. Kim, and S. C. Bai. 2013. Optimum feeding rates in juvenile olive flounder, Paralichthys olivaceus, at the optimum rearing temperature. Aquac. Nutr. 19:267-277.

Oyugi, D., J. Cucherousset, J. M. Ntiba, S. M. Kisia, D. M. Harper, and J. R. Britton. 2011. Life history traits of an equatorial carp Cyprinus carpio population in relation to thermal influences on invasive populations. Fish. Res. 110:92-97.

Ozorio, R. O. A., C. Andrade, V. M. F. A. Timoteo, L. E. C. Conceicao, and L. M. P. Valente. 2009. Effects of feeding levels on growth response, body composition, and energy expenditure in blackspot seabream, Pagellus bogaraveo, Juveniles. J. World Aquac. Soc. 40:95-103.

Person-Le, R. J., V. Buchet, B. Vincent, D. H. Le, and L. Quemener. 2006. Effects of temperature on the growth of Pollack (Pollachius pollachius) juveniles. Aquaculture 251:340-345.
Qiao, G., S. I. Park, and D. H. Xu. 2012. Clinical, hematological and biochemical alterations in Olive flounder Paralichthys olivaceus following experimental infection by Vibrio scophthalmi. Fish Aquat. Sci. 15:233-239.

Robbins, K. R., H. W. Norton, and D. H. Baker. 1979. Estimation of nutrient requirements from growth data. J. Nutr. 109:17101714.

Ronyai, A. and I. Csengeri. 2008. Effect of feeding regime and temperature on on-growing results of pikeperch (Sander lucioperca L.). Aquac. Res. 39:820-827.

Schulz, C., M. Huber, J. Ogunji, and B. Rennert. 2008. Effects of varying dietary protein to lipid ratios on growth performance and body composition of juvenile pike perch (Sander lucioperca). Aquac. Nutr. 14:166-173.

Turker, A. 2009. Effect of photoperiod on growth of trout (Oncorhynchus mykiss) in cold ambient sea water. Israel $\mathrm{J}$. Aquac. (Bamidgeh) 61:57-62.

Velmurugan, B., M. Selvanayagam, E. I. Cengiz, and E. Unlu. 2007. The effects of fenvalerate on different tissues of freshwater fish Cirrhinus mrigala. J. Environ. Sci. Health B. 42:157-163.

Van Ham, E. H., M. H. G. Berntssen, A. K. Imsland, A. C. Parpoura, B. S. E. Wenderlaar, and S. O. Stefansson. 2003. The influence of temperature and ration on growth, feed conversion, body composition and nutrition retention of juvenile turbot (Scohthalmus maximus). Aquaculture 217:547-558.

Wang, Y., L. J. Kong, K. Li, and D. P. Bureau. 2007. Effects of feeding frequency and ration level on growth, feed utilization and nitrogen waste output of cuneate drum (Nibea miichthioides) reared in net pens. Aquaculture 271:350-356.

Wang, N., X. Xu, and P. Kestemont. 2009. Effect of temperature and feeding frequency on growth performances, feed efficiency and body composition of pikeperch juveniles (Sander lucioperca). Aquaculture 289:70-73.

Wang, Y., J. Guo, K. Li, and D. P. Bureau. 2006. Effects of dietary protein and energy levels on growth, feed utilization and body composition of cuneate drum (Nibea miichthioides). Aquaculture 252:421-428.

Xia, J. and X. Li. 2010. Effect of temperature on blood parameters of the salamander Batrachupems tibetanus (Schmidt, 1925) (Amphibia: Hynobiidae). Russian J. Ecol. 41:102-106. 